\title{
Clinical Laboratory Educators' Conference 2016 Abstracts
}

\section{MINNEAPOLIS, MN}

The following abstracts were presented during the 2016 American Society for Clinical Laboratory Science (ASCLS) Clinical Laboratory Educators' Conference (CLEC) held February 25-27, 2016 in Minneapolis, MN. Abstracts were reviewed by appropriate representatives of the ASCLS Educational Scientific Assembly (ESA). The reviewers were the final authority in selecting or rejecting an abstract. (*-indicates presenter)

\section{Poster Presentations}

Self-Efficacy and Interprofessional Learning: An Exercise in Ethics for Health Science Students

Kathleen Cino PhD, RN, ${ }^{*}$ Rita Austin MS, MLS (ASCP) ${ }^{\mathrm{CM}} \mathrm{M}$, Cristina Casa RDH, MS, Adele Spencer $\mathrm{RDH}$, MS, Christine Nebocat MS, MLS (ASCP) ${ }^{\mathrm{CM}}$, Farmingdale State College School of Health Sciences, Farmingdale, New York

Interprofessional education (IPE) is becoming an increasingly important model for education in the fields of health sciences. An introductory Interprofessional Education exercise was developed for students in the Medical Laboratory Technology, Nursing and Dental Hygiene departments in the School of Health Sciences at Farmingdale State College. A 75 minute class was designed to include activities that encouraged analysis, comparisons and application of the ethical codes from each profession as a way to introduce students to the concept of Interprofessional education. Students were given a brief introduction to both IPE and professional ethics prior to completing the session activities. These were intended to highlight the shared values of each profession and to introduce and promote the principles of IPE. Interdisciplinary groups of students analyzed the ethical codes and noted similarities and differences prior to applying them to scenarios that demonstrated how ethics might impact professional practice. Students were assessed before and after the exercise using The Self-Efficacy for Interprofessional Experiential Learning (SEIL) scale developed by Mann, McFetridge-Durdle, Breau and Sarria (2012). The 16 items on the scale examine confidence levels related to different experiences associated with working with other professions. Results appear to show an overall increase in self-efficacy toward Interprofessional learning with some differences noted between the student groups.
There was an overall percent change of +6.5 when looking at student's understanding of the objectives of IPE, with a $15.8 \%$ change for Dental Hygiene, a $0.3 \%$ change for Medical Laboratory Technology and an $8.8 \%$ change for Nursing. Students were asked to comment on the session with only their discipline being reported. Qualitative analysis of the anonymous comments revealed that the majority of students concluded this was a positive learning experience. This exercise was successful in introducing the concepts of IPE through the study of ethics.

\section{Developing an Online Second Degree Clinical Laboratory Science Program \\ Lori Rice-Spearman, Ph.D., MT(ASCP); Wade Redman, Ph.D., MT(ASCP)DLM ${ }^{\mathrm{CM}}$; ${ }^{*}$ Tammy Carter, Ph.D., MT(ASCP); Joel Hubbard, Ph.D., MT(ASCP), Texas Tech University Health Sciences Center, Lubbock, Texas}

The purpose of this presentation is to provide the participants with information related to developing and delivering an online second degree Clinical Laboratory Science (CLS) program. The Texas Tech University Health Sciences Center School of Health Professions in 2007 sought to increase enrollment in the CLS program by developing an online second degree program for those individuals who earned Bachelor's degrees in a science field (biology, chemistry, etc.). Participants in this presentation will be able to describe the unique challenges associated with developing and delivering an online second degree CLS program and assess the resources required for positive student learning outcomes. The presenters will outline the process of developing the online second degree CLS program to include the following: Texas Higher Education Coordinating Board approval process, curriculum development, analysis of resources, delivery of lecture content, delivery of laboratory course work to ensure competency, and clinical education. In conclusion, an analysis and evaluation of outcome results for the online second degree CLS program was performed. For admissions, we receive an average of 125 applications and accept approximately 41 students, annually. A brief comparison of the ASCP board certification outcomes for online second degree CLS program and the traditional faceto-face CLS program shows a pass rate of $90 \%$ to $91 \%$, respectively. 


\section{CLINICAL PRACTICE}

The use of IF-AT (Immediate Feedback Assessment Test) in the classroom to improve student engagement

Cristine Fior Clemente dos Santos, PhD, MLS (ASCP) ${ }^{\mathrm{CM} S B B}$, Northern Illinois University

The objective of this exercise was to apply a learning system, called Immediate Feedback Assessment Test to promote a better engagement and content retention of students attending the class AHLS 471 (Molecular testing and Advance lectures) at MLS Program at NIU. A quiz of five points each, in the area of antibody identification was accessed.

IF-AT technique (Immediate Feedback Assessment Test) by Epstein Education Enterprises, is a learning system composed of an answer key in a rectangle scratch card marked with four types of answers, A, B, C or D. The correct answer for each question is marked with a "star". The students first take the test individually, and then the same test is taken in groups. The rule of this technique is that the group must agree with each answer before the card is scratched by a group leader. If the correct answer is chosen at the first time, the group earn 5 points, 3 points in the second try, 2 in the third, and no points after that. This technique was applied in a class of 24 students with a 5 questions quiz. The students were divided in 5 groups. Of the 24 students, $60 \%$ of their scores remained unchanged, $20 \%$ of the students increased their scores with the IF-AT technique and $20 \%$ lowered their scores. Overall, the IF-AT promoted better student engagement in the classroom. However, the average quiz scores were not improved by the majority of the students.

Impact of Time Lapse on ASCP Board of Certification Medical Laboratory Scientist (MLS) and Medical Laboratory Technician (MLT) Examination Scores

Karen A. Brown, MS, JoAnn P. Fenn, MS, "Vicki S. Freeman, PhD, Patrick B. Fisher, MA, Jonathan R. Genzen, MD, PhD, Nancy Goodyear, PhD, Mary Lunz Houston, PhD, Deedee O'Brien, BS, Geraldine Piskorski, MBA, Patricia A. Tanabe, MPA, ASCP Board of Certification Research and Development Committee

Chicago, IL

Problem: Research in several professional fields has demonstrated that delays (time lapse) in taking certification examinations may result in poorer performance by examinees. Thirteen U.S. states and/or territories require licensure for laboratory personnel. A core component of licensure is the successful completion of a certification examination. In addition, many facilities in non-licensure states either require certification for employment or preferentially hire certified individuals. Methods: The objective of this report was to analyze examinee performance on the American Society for Clinical Pathology (ASCP) Board of Certification (BOC) Medical Laboratory Scientist (MLS) and Medical Laboratory Technician (MLT) certification examinations to determine whether delays in taking the examination from the time of program completion were associated with poorer performance. Twenty months of examination data were obtained to look for changes in mean examination scaled scores and overall pass/fail rates. First time examinees (MLS, $\mathrm{n}=6,037$; MLT, $\mathrm{n}=3,920$ ) were divided into 3 month bins based on the interval of time between date of program completion and taking the certification examination. Results: Significant decreases in mean scaled scores and pass rates after the first quarter were observed in both MLS and MLT examinations for applicants who delayed taking their examination until the second, third, and fourth quarter after completing their training programs. Conclusions: Those who take the ASCP BOC MLS and MLT examinations are encouraged to do so shortly after completion of their educational training programs. Delays in taking an examination are generally not beneficial to the examinee and result in poorer performance on the examination.

\section{Assessing Student Stress in Clinical Laboratory Science} Programs: Online Versus Face-to-Face

Ericka Hendrix, Ph.D., MB(ASCP) ${ }^{\mathrm{CM}}$, Texas Tech University Health Sciences Center, Lubbock, Texas

Student stress is an issue that all clinical laboratory programs must address. The amount of stress that students perceive can impact grades, physical and mental health, and learning ability. Additionally, many programs have moved to online formats in which student stress is more difficult to detect. This study surveyed face-to-face clinical laboratory science students and online clinical laboratory students for perceived stress and coping mechanisms to determine differences in perceived stress and coping mechanisms. This study used a causal-comparative cross sectional design using inferential statistics to determine differences in perceived stress. Non-parametric tests were used to determine the differences in frequencies of coping mechanisms. Finally, correlational studies were conducted to determine if there was a relationship between student achievement (GPA) and perceived stress scores. The results of this study indicated that there was not a significant difference between online and traditional student perceived stress. Secondly, the results indicated that there was not a significant difference between face-to-face and online students use of coping mechanisms. Finally, the results of the study showed there was a significant correlation between perceived stress and GPA for student enrolled in the face-to-face program, but this was not the result for the online students. These results are useful for administrators 
and faculty to develop curricula that addresses stress, coping, and academic success for both online and face-toface clinical laboratory science programs.

Now Paging the Medical Laboratory Scientist to a Code Blue (A Simulation Training Experience)

*S. Renee Hodgkins, PhD, MT(ASCP) and Michael Waxman, M.D., The University of Kansas Medical Center, Kansas City, Kansas

Simulation training is gaining popularity as a method to educate health professionals on key competencies prior to performing a procedure on a real patient. For many health professionals, there are multiple opportunities to gain education through simulations with both mannequins and simulated patients (actors who are hired to play the role of the patient). Although clinical laboratory science educators have been "simulating" lab procedures in student labs for decades, the absence of laboratory students from simulation training with other health professionals is alarming. While 70-80\% of medical diagnoses are based on results of laboratory testing, medical laboratory scientists (MLS) are not utilized as a resource for information on test utilization and interpretation. Our MLS students were provided with the opportunity to be involved in an interprofessional Code Blue Simulation. We assessed attitudes towards teamwork, roles and responsibilities, communication, and simulation training using a modified Readiness for Interprofessional Learning Scale (RIPLS) both pre and post simulation. In this pilot study, students demonstrated increased confidence in their knowledge and improvement in their communication skills. MLS students were challenged in both defining their role in the code blue and providing active consultation on lab results. Attitudes assessed were sustained or increased post-simulation experience. All students involved felt that participating in the interprofessional simulation was a valuable experience. Overall, interprofessional simulation training experiences provide the MLS student with a unique opportunity to work in a healthcare team and gain confidence in their body of knowledge for providing laboratory information.

\section{Teaching Clinical Chemistry on a Shoestring Budget}

*Jan Hudzicki, Ph.D, MLS(ASCP) ${ }^{\mathrm{CM}}, \mathrm{SM}(\mathrm{ASCP})^{\mathrm{CM}}$

Jennifer Jones, MLS(ASCP) ${ }^{\mathrm{CM}}$, University of Kansas Medical Center, Kansas City, KS

The perceived need for expensive instrumentation often frustrates clinical chemistry lab instructors when planning lab activities. The variety of laboratory instrumentation encountered by our graduates makes it difficult and expensive to purchase instrumentation for a student lab. Minimal use of these instruments during the academic year results in malfunctions that require expensive service and repair. Our chemistry curriculum formerly consisted of a two course sequence, each having its own lab. Student evaluations indicated that there was excessive repetition of activities that did not translate to the real lab. As a result of these evaluations we revised our Clinical Chemistry curriculum by dropping one of the labs and creating a combined lecture/lab course, CLS 523, Fundamentals of Analytical Techniques. By focusing on the principles of chemical analyses used in a clinical chemistry lab (not on instrumentation), students gain an understanding of what is happening inside an instrument, which enhances critical thinking and troubleshooting skills. We will share our strategies for teaching this clinical chemistry lab using inexpensive assays that are spectrophotometer based and/or require minimal equipment yet introduce the fundamental concepts of the key methodologies of analytical chemistry and developing manual lab skills. We will discuss our rationale for the analytical methods we utilize in wet labs, methodologies that we discuss but do not performed, and the incorporation of lab operation skills into the course.

How a Leading Hospital Laboratory is Increasing Patient, Provider, and Payer Value Through Test Utilization Roberta (Bobbi) Jo Kochevar MBA, MLS(ASCP) ${ }^{\mathrm{CM}}$, North Memorial Health Care (NMHC), Robbinsdale, MN

The healthcare industry is shifting from a volume to valuebased payment model, ushering in new economic incentives to drive patient, provider, and payer behaviors. This shift requires a new set of management skills to encourage systems to align resources and improve outcomes \& efficiencies. NMHC, a leading integrated healthcare system, realized that adapting to this rapidly changing environment meant implementing a test utilization program to achieve four main priorities: population health, patient quality experience, operational efficiency, and profitable growth. Before, NMHC manually pulled analytics from multiple systems, resulting in strained resources, outdated results, and diminished service. NMHC leveraged the industry shift as an opportunity for advancement and implemented a healthcare relationship solution with real-time automation and data analytics to optimize communication while achieving $100 \%$ accountability. The interoperability provided immediate and accurate insight into client ordering behavior. Test utilization dashboards allow NMHC to pinpoint which test(s) are over-and underutilized. In one-month, NMHC identified an unnecessary test, launched proper communication channels, and monitored the discontinued test. These analytics allow NMHC to partner with providers on trends by specialty and demographic. Previously, this would have been impossible to detect or monitor. Ordering analytics are also compared with reimbursement rates to determine 
profitability trends. Pathologists can collaborate with providers to identify proper tests for patients based on publications, analytics, reimbursement rates, and benchmark comparisons. NMHC needed access to the right information at the right time to sustain. Real-time interoperability allows providers, patients, and payers to fully engage in an efficient and accurate manner.

Increasing the Value of Undergraduate Clinical Laboratory Science Programs in Academic Institutions Through Undergraduate Research Programs

William J. Korzun, Ph.D., MT(ASCP), Virginia Commonwealth University, Richmond, VA

Clinical Laboratory Science (CLS) Programs in academic institutions are under increasing pressure to engage in scholarly activities, obtain grants to support them, and generate external awareness of the institution's status as a research-intensive university. A few years ago, we discovered that many of our undergraduate students, after completing two semesters in our CLS Program, possessed the lab skills that were highly desirable to researchers in other academic units. Consequently, the Department of Clinical Laboratory Sciences, in the School of Allied Health Professions, began participating in, and has successfully received funding from the Undergraduate Research Opportunities Program at Virginia Commonwealth University. This fellowship program provides outstanding undergraduates the chance to make substantial and significant progress on a structured research endeavor during the summer in collaboration with a faculty mentor. These activities have increased the visibility of research efforts within the department and student motivation to complete advanced degrees with research requirements. Outcomes have included presentations at university-wide research symposia, presentations at state professional meetings, co-authorship on peer-reviewed publications, increased requests from the School of Medicine for research collaboration with the CLS Dept., subsequent enrollment in graduate programs, and recognition of departmental accomplishment of the institution's research mission. We have concluded that the investment of faculty time and effort to mentor undergraduate students who are interested in research, has led to an identity for the Department of Clinical Laboratory Sciences as a valuable component of the School of Allied Health Professions and Virginia Commonwealth University.

The Use of a Case Study Approach to Recruit High School Students with an Interest in Healthcare to Medical Laboratory Sciences and other Allied Health Professions Kristin Landis-Piwowar, Ph.D., MLS(ASCP) ${ }^{\mathrm{CM}}$, Oakland University, Rochester, MI
Medical Laboratory Science educators continually strive to adopt effective techniques that yield both improved understanding of and tools to recruit students to the profession. Macomb Community College, in partnership with Oakland University, offers a four-day summer camp entitled, "Healthcare Professions Camp," in which high school students explore health related professions. The Oakland University, Biomedical Diagnostic and Therapeutic Sciences Program (BDTS) offers Bachelor of Science degrees in Medical Laboratory Science, Histotechnology, Nuclear Medicine Technology and Radiologic Technology and is thus an important contributor to this event. The BDTS program provides a full day of activity for the camp. The experience is designed to mimic the examination of a single patient who is evaluated in different departments of a hospital and, most notably, different areas of the clinical laboratory. The camp participants are escorted through the "radiology department" where they encounter radiologic technologists and nuclear medicine technologists and to the "clinical pathology department" where they perform procedures in immunohematology, urinalysis, microbiology, and hematology. At the end of the day, the participants compile all of the data that was collected on the patient, assess the significance of their findings, and discuss the importance of the numerous individuals that were involved in the patient's diagnosis. While the case and some of the specimens are not real, the camp participants leave the camp with a new perspective on healthcare professions and note that their experience was positive and impactful.

The Effect of Phlebotomist Patient-Centeredness on Emergency Department Patients' Experience of Care

Stephen Aragon, PhD; ${ }^{*}$ Lisa Maness, PhD, Winston Salem State University, Winston-Salem, NC, Laura McGuinn, MD, University of Oklahoma, Oklahoma City, OK, Sabina Gesell, PhD, Wake Forest University, Winston-Salem, NC

PROBLEM: Little is known about the patient-centeredness of phlebotomists or its influence on patients' experience of care. The aims of this investigation were to determine if: phlebotomist patient-centeredness influenced emergency department (ED) patients' experience of care, by how much, and if the influence was robust across samples. DESIGN: A two-factor multigroup structural equation modeling design was employed, with asymptotic distribution free estimation, and three national random samples of ED patients. The hypothesized phlebotomist patient-centeredness model was subjected to goodness of fit, measurement invariance, and competing model experiments. RESULTS: Evidence supported the hypothesized model's fit and robustness across samples $\left(\chi^{2}\right.$ $(21)=22.92, p=.35 ; \chi^{2} / d f=1.09$; RMSEA $=.01, p=1.00$, $\mathrm{SRMR}=.031, \mathrm{CFI}=.99)$. It was further supported by small 
standardized residual covariances $(<2.0)$. Effect sizes were directionally appropriate, large, and significant $(\mathrm{p}<.001)$. The patient-centeredness of phlebotomists explained 44 percent of the variance of ED patients' experience of care, $\left(\mathrm{CI}_{90}=.351-.513, \mathrm{p}=.004\right)$. The model was sustained when compared to a competing model $\left(\chi^{2} \Delta(18)=19.82, p\right.$ $=.34)$. The total standardized effects of phlebotomist patient-centeredness on experience of care, ratings of care, and likelihood to recommend the ED were $.663, .604$, and .654 respectively. IMPLICATIONS: The results reflect the positive influence of phlebotomist patient-centeredness on patients' experience of care and potential for improving quality. This investigation offers an evidence-based phlebotomist patient-centeredness model for improving quality.

Using Case Studies as a Tool to Teach Laboratory Techniques and Principles in a Clinical Molecular Diagnostics Laboratory

${ }^{*}$ Michelle Russell, M.S., MLS (ASCP) ${ }^{\mathrm{CM}}$, Catherine Dixon, MLS(ASCP) ${ }^{\mathrm{CM}}$, University of Michigan Health System, Ann Arbor, Michigan

Our academic hospital serves as a clinical affiliate for various NAACLS accredited MLS university programs. In the past, providing students with interactive bench training in our molecular diagnostics laboratory rotation has been challenging. As such, student surveys have noted their dissatisfaction in the molecular diagnostics portion of their internship. Reasons for the challenges faced include the imperative accuracy required in molecular oncology testing, high cost of molecular reagents, limited rotation length, and lack of interactive learning activities. To address this problem, we proposed a concise rotation through the molecular laboratory using a case study teaching approach. Three case studies centering on lung carcinoma, acute myeloid leukemia, and familial hyperlipidemia were developed to emphasize various clinical and technical aspects of the molecular diagnostics laboratory. Each case study included relevant background information, learning objectives and activities, and followup questions. Students performed assays under direct supervision using dedicated reagents and samples. A daily schedule was implemented as a tool to provide structure to students and instructors. Laboratory directors and technical specialists were recruited to discuss the various technical aspects and clinical impact of molecular oncology testing. Following the first year of this approach, student surveys have demonstrated a greater satisfaction in the molecular diagnostics rotation as evidenced by improved positive ratings and comments, bringing our lab in line with the other hospital laboratory rotations. This new strategy has also served as the foundation for a molecular diagnostics internship program.
Make it Stick, Methods for Improving Student Learning ${ }^{*} J a n e t$ Oja MLS(ASCP) ${ }^{\mathrm{CM}}$, Scott Wright $\mathrm{M}(\mathrm{ASCP})^{\mathrm{CM}}$, Kenton Cummins BS(ASCP) ${ }^{\mathrm{CM}}$, Medical Laboratory Sciences, Weber State University, Ogden, UT

As educators, we are constantly striving for ways to help our students be more effective learners. Much of what we teach first requires efficient memorization and recall before a student can develop higher level decision making skills required in the clinical laboratory. Beginning spring semester 2015, three WSU MLS faculty implemented techniques described by Peter Brown in his book titled "Make it Stick, the Science of Successful Learning." These techniques include: daily in-class quizzes, interleaving, spacing, and reflections. Daily in-class quizzing provides the students with questions to engage the mind at the beginning of lecture, grants longer attention span, and allows them to have more 'light-bulb' moments during lecture. Interleaving is the action of bringing more than one subject into a concept. Spacing provides the students with sufficient time to forget material, and to recall them once again. Reflections force the student to form concepts into their own words, causing long-term cognitive imprinting of important scientific doctrine. Each of these techniques require students to access their memory and retrieve information in different ways, effectively developing long term memory. After implementing these techniques, major quiz and test scores from six MLS courses have consistently risen by $1-2 \%$ when compared to the past four years. In addition to improved test scores, faculty have observed an increase in both student confidence and laboratory performance. We are hoping the make it stick techniques will also result in higher ASCP certification exam scores.

\section{Interprofessional Education: Making it Work for the Laboratory Educator}

Kay Rasmussen, MS, MLS(ASCP) ${ }^{\mathrm{CM}}$, University of South Dakota, Vermillion, SD

Properly trained healthcare teams communicate more effectively and participate in medical processes with an overall result of higher-quality patient care (Delunas \& Rouse, 2014). Healthcare institutions are turning to higher education partners to prepare and develop practitioners who embrace collaborative working models. The University of South Dakota's School of Health Sciences (SHS) has adopted and practices an all-inclusive interprofessional education (IPE) curriculum across its healthcare disciplines, which includes medical laboratory science. The School uses theoretical applications and practical simulations to promote dialogue and best practice decisions in the context of interprofessional care. By example, MLS students recently reflected upon their developing expertise and role as members of the healthcare 
team following a practical simulation specific to transfusion medicine with physician assistant partners. The MLS students' pre/post transfusion medicine examinations showed a minimum of $10 \%$ change in subject comprehension. Another example of an IPE activity aimed at measuring student attitude change is the SHS interprofessional team training event that occurs each fall. This event stresses the importance of interprofessional collaboration rather than discipline specific knowledge. Students complete a pre-event self-assessment. Students are randomly selected and assigned to health care teams. Each team is comprised of a student from the 10 different disciplines. Following the exercise, students take a post selfassessment. Time after time, the post assessments revealed students leave the exercise with positive feelings about what they accomplished for patients when working as a member of a health care team and the students report being more comfortable working with each other.

\section{The Positive Impact on Student Learning in "Flipped" Clinical Instrumentation Course}

${ }^{*}$ Kyle B Riding, PhD, MLS(ASCP) ${ }^{\mathrm{CM}}$, Keiser UniversityOrlando, Florida Campus, ${ }^{*}$ Brenda Berube, MS, Ed.S. MT(ASCP), School District of Osceola County, Florida

Educational research supports that the flipped approach promotes an increase in student engagement and advanced critical questioning of scientific principles. This case study explores the application of the flipped classroom approach to the redesign of a traditionally-delivered clinical instrumentation course. A persistent barrier of the course was that students struggled with applying scientific principles to problem-based scenarios in topics of photometry, electrochemistry, immunochemistry and separation techniques. Instructors reported the absence of a companion laboratory course as a barrier to students succeeding at application-based assignments. The flipped classroom delivery model was designed with weekly activities that were focused on problem-based scenarios, laboratory demonstrations, and multiple weekly quizzes. Additionally, journaling allowed for a reflective assignment to enhance meta-cognition. The variety of learning experiences could not be conducted under the time restraints of a traditionally-delivered course. Appropriate scaffolding of assignments allowed students to achieve statistically similar $(\mathrm{p}=0.4683)$ average scores between recall-based pre-face-to-face quizzes and application-based post-face-to-face quizzes. The average mid-term and final exams scores (means of 88 and 80 , respectively) were higher than expected - indicating a higher level of understanding about the material. Downsides include less in-person interaction with the faculty and more advanced prep and planning on the part of the instructor. In conclusion, the flipped classroom model was successfully used in this course to overcome barriers related to critical-thinking and lack of laboratory exposure as it relates to the course material.

Case Presentations as a Foundation for Student-Centered Learning of Laboratory Medicine in a Physician Assistant Curriculum

George B. Kudolo, PhD, FACB and *Linda A. Smith, PhD, MLS(ASCP) ${ }^{\mathrm{CM} B B}$, University of Texas Health Science Center, San Antonio, TX

Although little time is devoted to instruction in interpretation of clinical laboratory tests, physician assistants (PAs) may have to personally interpret test results whereas radiology and anatomic tests are interpreted for them. The CLS faculty, with input from PA faculty, developed a laboratory medicine course. Recently, in addition to didactic instruction, case study development was instituted to enhance students' learning and retention. Early in the semester, the class was divided into groups of five students and assigned case topics ranging from tumor markers to endocrinology. The cases were designed to have students learn to integrate clinical and laboratory information and present an overall picture of the patient's disease as well as practice sharing information with colleagues. Students were given guidelines but had the opportunity to develop role play. Each group developed and delivered a 15-minute presentation including a skit by one group. They also supplied two questions/group for the final examinations. Student attitudes towards this approach were positive and course evaluation was 4.6/5.0 with an $87 \%$ response rate. Typical student comments included: "...group project was a great experience. I would not change anything..." "....gained a new found respect for the [CLS] profession. " "...I will use what I have learned in this class on a daily basis as a practicing PA." "It is important to know what labs to order, what we are looking at and why. "The combination of student-developed cases and didactic lectures is an effective way to teach interpretation of laboratory tests to PAs.

\section{Interprofessional Education in the Health Profession of Clinical Laboratory Sciences}

Jose H. Salazar, PhD, MLS (ASCP) ${ }^{\mathrm{CM}}$, The University of Texas Medical Branch, Galveston, Texas

Maintaining and increasing the quality of health care will require multiple health professions working together to meet the needs of the public. Interprofessional collaboration between the health professions has been recommended as an avenue to develop and support effective and efficient teams of health professionals. The lack of interprofessional collaboration can lead to increased healthcare costs, inpatient hospitalization, patient 
mortality, and low quality of patient care. Interprofessional education (IPE) is a critical component of a health professions education curriculum in addressing the need for interprofessional collaboration in professional practice. Unfortunately, IPE is not currently embedded into the health professions curricula. The purpose of this qualitative exploratory case study was to explore and describe how clinical laboratory science students at a tertiary university hospital perceive and conceptualize IPE through exposure to IPE in clinical preceptorship and service learning experiences. The data collection and analysis process included multiple interviews, student clinical preceptorship journals, student service learning journals, and a researcher's journal. The constant comparison method (Glaser \& Strauss, 1967) was used throughout the study as themes and patterns emerged from the raw data and were discovered. Findings indicated that participants believed that IPE helped reduce professional hierarchy, promoted equality and respect and maximized interprofessional collaboration between the health professions. The results from this study reveal how an interprofessional curriculum can unite the health professions early in their formative years to address the inefficiencies and ineffectiveness of a disjointed health care team that negatively affects health care in the United States today.

\section{Effects of the Clinical Laboratory Initiative to Mentor Baccalaureate Students Grant}

${ }^{*}$ Rajkumar Rajendran, MS, MLS (ASCP) ${ }^{\mathrm{CM}}$, *Julie Soder, MS, MLS (ASCP) ${ }^{\mathrm{CM}}$, Vicki Freeman, PhD, MLS (ASCP) ${ }^{\mathrm{CM}}$, Chantele Singleton MBA, Shayne Washington, Med, University of Texas Medical Branch, Galveston, Texas

Problem: The purpose of the Clinical Laboratory Initiative to Mentor Baccalaureate Students (CLIMBS) grant's was to successfully train individuals who were either unemployed or underemployed so they could obtain a certification with American Society for Clinical Pathology (ASCP) and find employment. CLIMBS students were given access to services including training, tutoring, a laptop, counseling, books, scrubs, and health insurance. The primary purpose of this study will examine the success of the student's training to determine if the additional resources provided by the grant made a positive contribution to the student's success. Design/Method: This study is a cross-sectional survey which includes 140 students which completed the program from August of 2013 to August of 2015. Aim 1 will examine students' entering grade point average (GPA) compared to their final GPA to determine if there is a statistical difference using t-test at $\alpha 0.05$. Aim 2 will determine if there was a statistical significance between the number of unemployed or underemployed individuals before and after training utilize chi-square $\alpha$ 0.05. Aim 3 will compare the mean salaries difference of the CLIMBS participants before and after training. Results: There was a statistical difference in the employment status and the mean salary before and after training. Conclusion: Overall the CLIMBS grant had a positive impact on student outcomes.

Masters in Clinical Laboratory Science at University of Texas Medical Branch, a Success Story

${ }^{*}$ Jianzhi Zhang, MD \& Muneeza Esani, MHA, University of Texas Medical Branch, Galveston, TX

The goal of Clinical Laboratory Science (CLS) Master's program is to provide quality laboratory practitioners with skills in applying Evidence-based Laboratory Science and leadership to benefit the patients and profession that they serve and to contribute to the scientific knowledge in CLS. The University of Texas Medical Branch introduced three new Master's degree programs in CLS in 2011. This program consists of three tracks which include a Masters in science for students with no CLS background, a distance Master's in science for individuals who already hold a bachelor's degree in CLS and a distance Master's in Science in Transfusion Medicine. The program started with 5 students in 2011 and we currently have 35 Masters Students between the three tracks. This program will have graduated 23 students by the end of year 2015. Students complete graduate level clinical laboratory science courses while in the program. The culminating experience of the master program in clinical laboratory science is the completion of a thesis. The intent and construct of a thesis varies by research project area and its supervision is handled by a member of CLS Faculty as a thesis chair, a course instructor and the Master's thesis committee consisting of CLS faculty members. Distance students are allowed to also have a research mentor at their clinical facility. Graduate satisfaction and professional advancement indicate that CLS Master's program is a success story. This poster will focus on the design and implementation of a CLS Master program and will also include graduate success stories.

A Student-Centered Active Learning Approach to Teaching Anemias in a Medical Laboratory Science Hospital-based Program

${ }^{*}$ Ashley Zawacki, MS, MLS(ASCP) ${ }^{\mathrm{CM}}$, Marianne Knutson, MBA, MT(ASCP), Hennepin County Medical Center Minneapolis, MN, Elaine M. Keohane, $\mathrm{PhD}$, MLS(ASCP) ${ }^{\mathrm{CM}}$, Rutgers, The State University of New Jersey, Newark, NJ

The traditional education model involves a teachercentered environment with students playing a passive role in the education process. An environment where active 


\section{CLINICAL PRACTICE}

learning is fostered promotes student exploration of material and more student engagement and comprehension, in turn improving critical thinking and problem solving skills. An active-learning model, the flipped classroom, was developed and implemented for didactic and student lab instruction in anemias in a Medical Laboratory Science (MLS) hospital-based program. For pre-workshop and pre-laboratory components narrated PowerPoint presentations were developed to provide background knowledge. While a traditional PowerPoint was used as backbone for each in-class workshop and laboratory session, all are filled with critical thinking questions, assignments, and interactive breakout activities such as case studies, role-playing, games, or mini student presentations. These activities reinforce concepts and provide opportunities for interaction among students. Alumni and MLS educators $(n=21)$ who viewed the materials strongly agreed (81\%) and agreed (19\%) that the in-class workshops achieved an appropriate balance of activities and student interaction with formal lecture material. In initial student surveys, $83 \%$ strongly agreed that the pre-workshop content was helpful in understanding material covered in class, that the in-class activities helped to further understand the content, and that interaction with other students was a helpful way to learn. Only 66\% strongly agreed, 17\% agreed, and 17\% disagreed that the flipped classroom was a better way to learn compared to traditional lectures. Additional modules will be implemented and evaluated to further assess the effectiveness of this model.

\section{Technology Demonstrations}

Active Learning through Development of Vignettes and Pre-Laboratory Assessments for Hematology Laboratory Instruction

${ }^{*}$ Candice Grayson, MS, MLS (ASCP) ${ }^{\mathrm{CM}}$, Community College of Baltimore County, Baltimore, MD, Nadine
Fydryszewski, PhD, MLS, Rutgers University, Newark, NJ

With a growing trend of less instructional time and more skills required to be taught, alternative educational tools and models need to be evaluated for effectiveness and utilization within the curriculum. The current educational trend in the medical field focuses on the use of simulation in patient care scenarios; however, in the clinical laboratory sciences simulation or alternative educational methodologies incorporating pre-laboratory psychomotor skills requirements has not been thoroughly researched or designed. The goal of this project was to develop prelaboratory simulation modules for one core medical laboratory science clinical course, hematology. This pilot model would serve as a template to develop additional simulation exercises for the other clinical courses. In an effort to encourage active learning in medical laboratory technician students, vignettes and pre-laboratory modules were created. These simulation modules are not replacing in-class or traditional laboratory instruction, but are supplementing education as pre-laboratory preparation and review prior to clinical rotations. Students are required to preview the laboratory skills simulation videos and complete the self-assessments prior to in-class lab sessions and, as a refresher before beginning clinical rotations. Student feedback via an informal survey from one class in the medical laboratory technician program $(n=14)$ was positive and interestingly enough they found mistakes or ways that the modules can be improved, indicating their interest in seeing this project continue. The students' comments were that the mobility and access to the instructor's step-by-step procedure of the vignettes helped to prepare them prior to entering the laboratory. The selfassessments within the modules were non-graded and were quick checkpoints for the students, with feedback and direction of where to review if incorrect.

\section{Editor-in-Chief Position}

Applications for editor-in-chief (EIC) for Clinical Laboratory Science are now being accepted. The EIC provides leadership and direction that results in the publishing of a well-respected, peer-reviewed, scientific journal. The EIC develops and reviews manuscripts, organizes journal functions to maintain editorial integrity and evaluates and makes adjustments to the journal as appropriate. Applicants should be a member of ASCLS, have authored peer reviewed publications, and served as a section editor of the journal or a similar position with another journal. Clinical Laboratory Science is the official journal of the American Society for Clinical Laboratory Science (ASCLS). The journal is published quarterly by Westminster Publishers and is listed in PubMed. Application can be made vie email by September 1, 2016 to ASCLS President-Elect Suzanne Campbell (suzanne.campbell@hotmail). 